\title{
On a Generalization of Meyniel's Conjecture on the Cops and Robbers Game
}

\author{
Noga Alon * \\ Tel Aviv University \\ and \\ Institute for Advanced Study, Princeton \\ nogaa@tau.ac.il \\ Abbas Mehrabian \\ Department of Combinatorics and Optimization \\ University of Waterloo \\ amehrabian@uwaterloo.ca \\ Submitted: Oct 11, 2010; Accepted: Jan 11, 2011; Published: Jan 19, 2011 \\ Mathematics Subject Classification: 05C57
}

\begin{abstract}
We consider a variant of the Cops and Robbers game where the robber can move $s$ edges at a time, and show that in this variant, the cop number of a connected graph on $n$ vertices can be as large as $\Omega\left(n^{\frac{s}{s+1}}\right)$. This improves the $\Omega\left(n^{\frac{s-3}{s-2}}\right)$ lower bound of Frieze et al. [5], and extends the result of the second author [10], which establishes the above bound for $s=2,4$.
\end{abstract}

\section{Introduction}

The game of Cops and Robbers, introduced by Nowakowski and Winkler [11] and independently by Quilliot [13], is a perfect information game played on a finite graph $G$. There are two players, a set of cops and a robber. Initially, the cops are placed on vertices of their choice in $G$ (where more than one cop can be placed at a vertex). Then the robber, being fully aware of the cops placement, positions herself in one of the vertices of $G$. Then the cops and the robber move in alternate rounds, with the cops moving first, where every cop may move in each round and players are permitted to remain stationary on their turn if they wish. The players use the edges of $G$ to move from vertex to vertex. The cops

${ }^{*}$ Research supported in part by an ERC Advanced grant, by a USA-Israeli BSF grant, by the Oswald Veblen Fund and by the Bell Companies Fellowship. 
win and the game ends if eventually a cop steps into the vertex currently occupied by the robber; otherwise, i.e., if the robber can elude the cops indefinitely, the robber wins. The parameter of interest is the cop number of $G$, which is defined as the minimum number of cops needed to ensure that the cops can win. We will assume that the graph $G$ is simple and connected, because deleting multiple edges or loops does not affect the set of possible moves of the players, and the cop number of a disconnected graph obviously equals the sum of the cop numbers for each connected component.

For a survey of results on the cop number and related search parameters, see [6]. The best known open question in this area is Meyniel's conjecture, published by Frankl [4]. It states that for every graph $G$ on $n$ vertices, $O(\sqrt{n})$ cops are enough to win. This is asymptotically tight, i.e. for every $n$ there exists an $n$-vertex graph with cop number $\Omega(\sqrt{n})$. The best upper bound found so far is $n 2^{-(1-o(1)) \sqrt{\log _{2} n}}([5,7,14])$.

Here we consider the variant where in each move, the robber can take any path of length at most $s$ from her current position, but she is not allowed to pass through a vertex occupied by a cop. The parameter $s$ is called the speed of the robber. This variant was first considered in [3]. Frieze et al. [5] showed that the cop number of a connected $n$-vertex graph can be as large as $\Omega\left(n^{\frac{s-3}{s-2}}\right)$. Later, the second author improved the lower bound to $\Omega\left(n^{\frac{s}{s+1}}\right)$ for $s=2,4[10]$. In this note we show that this lower bound holds for all $s$.

\section{The main result}

Let $k$ be a positive integer. For a vertex $u$ of a graph $G, N_{k}(u)$ denotes the set of vertices whose distance from $u$ is exactly $k$. If $k=1$, then we simply write $N(u)$. If $A$ is a subset of vertices, then $N_{k}^{A}(u)$ denotes the set of vertices $v$ such that:

- The distance between $u$ and $v$ is $k$, and

- for every shortest $(u, v)$-path $u u_{1} u_{2} \ldots u_{k-1} v$, we have $u_{1} \notin A$.

Note that for every $u, A$ and $k, N_{k}^{A \cap N(u)}(u)=N_{k}^{A}(u)$. For vertices $u, v$, we denote their shortest-path distance by $d(u, v)$. The diameter of $G$ is the maximum distance between any two vertices of $G$.

Lemma 1. Let $s, d, m$ be positive integers and $q$ be a positive real such that $q d^{s} / 2$ is an integer larger than $m$. Let $G$ be a d-regular bipartite graph of diameter larger than $s$ with the following properties:

(1) For every two vertices $u, v$ of $G$ of distance at most $s+1$, there are at most $m$ distinct shortest $(u, v)$-paths.

(2) For every vertex $u$ of $G$ and every subset $A$ of size at most $m,\left|N_{s}^{A}(u)\right| \geq q d^{s}$.

Then, assuming the robber has speed $s$, the cop number of $G$ is at least $q^{2} d^{s} / 24 m s$. 
Proof. Let us first define a few terms. A cop controls a vertex $u$ if the cop is on $u$ or on an adjacent vertex. A cop controls a path if it controls a vertex of the path. The cops control a path if there is a cop controlling it. A vertex $r$ is safe if there is a subset $X \subseteq N_{s}(r)$ of size $q d^{s} / 2$ such that for all $x \in X$, all shortest $(r, x)$-paths are uncontrolled.

Let the number of cops be $c$ with $c<q^{2} d^{s} / 24 m s$, and we will show that the robber can evade forever. If this many cops can capture the robber, then they can capture her from any starting configuration. Thus we may assume that the cops all start in one vertex $u$, and the robber starts in a vertex $r$ at distance $s+1$ from $u$. Such two vertices exist as $G$ has diameter larger than $s$. Property (2) gives $N_{s}(r) \geq q d^{s}$, and by property (1), the cops control at most $m$ vertices of $N_{s}(r)$. Since $q d^{s}-m>q d^{s} / 2$, the robber is in a safe vertex at the starting configuration. Hence we just need to show that if the robber is in a safe vertex before the cops move, then she can move to a safe vertex after the cops move.

Suppose that the robber is in a safe vertex $r$, so by definition, there is a subset $X \subseteq N_{s}(r)$ of size $q d^{s} / 2$ such that for all $x \in X$, all shortest $(r, x)$-paths are uncontrolled. Denote by $A$ the set of vertices of all shortest $(r, x)$-paths for all $x \in X$. In particular, $r \in A$ and $X \subseteq A$. Now, the cops move to new positions. At this moment there is no cop in $A$, so the robber is able to move to any vertex of $X$ in her turn; thus to complete the proof, we need to show that there is a safe vertex in $X$.

Claim. Every vertex $u \notin A$ has at most $m$ neighbors in $X$.

Proof. If $u$ has no neighbor in $X$, then the claim is true, otherwise let $x \in X$ be adjacent to $u$. Note that as $d(r, x)=s$, we have $d(r, u) \in\{s-1, s, s+1\}$. The graph $G$ is bipartite, so $d(r, u) \neq s$. If $d(r, u)=s-1$ then $u$ is on a shortest $(r, x)$-path, which contradicts the assumption $u \notin A$. Therefore, $d(r, u)=s+1$, and $x$ is on a shortest $(r, u)$-path. Hence by property (1), the number of neighbors of $u$ in $X$ is at most $m$.

Remark. It can be shown using a similar argument that every $x \in X$ has at most $m$ neighbors in $A$.

By an escaping pair we mean a pair $(x, y)$ of vertices with $x \in X$ and $y \in N_{s}^{A}(x)$. We call $x$ the head and $y$ the tail of the pair. By the remark, the set $A \cap N(x)$ has at most $m$ elements, and property (2) ensures that $\left|N_{s}^{A}(x)\right|=\left|N_{s}^{A \cap N(x)}(x)\right| \geq q d^{s}$. That is, every $x \in X$ is the head of at least $q d^{s}$ distinct escaping pairs. We say that an escaping pair $(x, y)$ is free if all shortest $(x, y)$-paths are uncontrolled. We just need to prove that there is an $x \in X$ such that $x$ is the head of at least $q d^{s} / 2$ free escaping pairs, because then $x$ would be a safe vertex, and the robber, having speed $s$, can move to $x$ in her move. If $(x, y)$ is an escaping pair, then every shortest $(x, y)$-path is called an escaping path. By definition, every escaping path can be written as $u_{1} u_{2} u_{3} \ldots u_{s+1}$, where $u_{1} \in X$ and $u_{2} \notin A$.

Claim. Each cop controls at most $3 m s d^{s}$ escaping paths.

Proof. We first prove that every vertex $v$ is on at most $d^{s}+m s d^{s-1}$ escaping paths, and if $v \notin X$, then $v$ is on at most $m s d^{s-1}$ escaping paths. Let $u_{1} u_{2} u_{3} \ldots u_{s+1}$ be an escaping path with $u_{1} \in X$ and $u_{2} \notin A$, such that $v$ is its $i$-th vertex, i.e. $v=u_{i}$. 
Assume first that $i \neq 1$. There are at most $d$ choices for each of $u_{i-1}, \ldots, u_{2}$, and for each of $u_{i+1}, u_{i+2}, \ldots, u_{s+1}$. By the previous claim, once $u_{2}$ is determined, there are at most $m$ choices for $u_{1}$. Consequently, for each $2 \leq i \leq s+1, v$ is the $i$-th vertex of at most $m d^{s-1}$ escaping paths, so if $v \notin X$ then $v$ is on at most $m s d^{s-1}$ escaping paths.

If $i=1$ then $v \in X$ and there are at most $d$ choices for each of $u_{2}, u_{3}, \ldots, u_{s+1}$, thus each $v \in X$ is the first vertex of at most $d^{s}$ escaping paths. This shows that $v$ is on at most $d^{s}+m s d^{s-1}$ escaping paths.

Recall that since the robber was in a safe vertex before the cops' move, no cop is in $A$ at this moment. By the previous claim, each cop controls at most $m$ vertices of $X$, through which he can control at most $m\left(d^{s}+m s d^{s-1}\right)$ escaping paths. Through every other neighbor he can control at most $m s d^{s-1}$ escaping paths. He controls $d+1$ vertices in total, so he controls no more than

$$
m\left(d^{s}+m s d^{s-1}\right)+(d+1-m)\left(m s d^{s-1}\right) \leq 3 m s d^{s}
$$

escaping paths.

Since there are $c$ cops in the game, the cops control at most $3 m s d^{s} c$ escaping paths. By controlling each escaping path, the cops can decrease the number of free escaping pairs by at most 2 (as each path has two endpoints), hence the number of non-free escaping pairs is at most $6 m s d^{s} c$.

Now we prove that there is an $x \in X$ such that $x$ is the head of at least $q d^{s} / 2$ free escaping pairs, completing the proof. Recall that every $x \in X$ is the head of at least $q d^{s}$ escaping pairs. Hence if there were no $x \in X$ such that $x$ is the head of at least $q d^{s} / 2$ free escaping pairs, then every $x \in X$ would be the head of at least $q d^{s} / 2$ non-free escaping paths. As by definition of safeness, $X$ has size $q d^{s} / 2$, this would imply that the number of non-free escaping pairs is at least $\left(q d^{s} / 2\right)^{2}$, which is larger than $6 m s d^{s} c$. This contradiction shows that the robber can evade $c$ cops forever.

Let $k, s$ be positive integers and $d=2^{k}$. Let $x_{1}, x_{2}, \ldots, x_{d}$ be the $d$ elements of $G F\left(2^{k}\right)$ represented as column vectors of length $k$ over $\mathbb{Z}_{2}$. Let $H$ be the following $1+k(s+1)$ by $d$ matrix over the field $\mathbb{Z}_{2}$ :

$$
H=\left[\begin{array}{cccc}
1 & 1 & \ldots & 1 \\
x_{1} & x_{2} & \ldots & x_{d} \\
x_{1}^{3} & x_{2}^{3} & \ldots & x_{d}^{3} \\
\vdots & \vdots & \ddots & \vdots \\
x_{1}^{2 s+1} & x_{2}^{2 s+1} & \ldots & x_{d}^{2 s+1}
\end{array}\right]
$$

Let $S=\left\{e_{1}, e_{2}, \ldots, e_{d}\right\} \subseteq \mathbb{Z}_{2}^{1+k(s+1)}$ be the set of columns of $H$. It is known that every set of $2 s+3$ columns of $H$ is linearly independent over $\mathbb{Z}_{2}$ (c.f., e.g., [1]), hence, in particular, every $(2 s+2)$-subset of $S$ is linearly independent over the field $\mathbb{Z}_{2}$. Let $G$ be the graph with vertex set $\mathbb{Z}_{2}^{1+k(s+1)}$, and with vertices $u, v$ adjacent if $u-v \in S$ (the Cayley graph of the additive group $\mathbb{Z}_{2}^{1+k(s+1)}$ with respect to $S$ ). 
Lemma 2. If $d \geq 2(s+1)$ !, then the graph $G$ has the following properties.

(i) G is connected.

(ii) $G$ is d-regular.

(iii) $G$ is bipartite.

(iv) For every two vertices $u, v$ of $G$ of distance at most $s+1$, there are at most $(s+1)$ ! distinct shortest $(u, v)$-paths.

(v) For every vertex $u$ of $G$ and every subset $A$ of size at most $(s+1)$ !, $\left|N_{s}^{A}(u)\right| \geq$ $(d / 2 s)^{s}$.

(vi) G has diameter larger than $s$.

Proof. (i) To show connectivity one has to prove that every element of $\mathbb{Z}_{2}^{1+k(s+1)}$ can be written as a linear combination of members of $S$, which is equivalent to the matrix $H$ having rank $1+k(s+1)$. Note that $H$ has $1+k(s+1)$ rows, thus we need to show that no nontrivial linear combination of its rows over $Z_{2}$ is the zero vector. But it is known that the rows $2,3, \ldots, 1+k(s+1)$ generate a dual $\mathrm{BCH}$ code, and every nontrivial linear combination of them has almost the same number of zeros and ones (see [9]).

(ii) This is clear as $|S|=d$.

(iii) This follows from the fact that each member of $S$ has 1 as its first coordinate, hence there is no odd-size subset of $S$ whose sum of members is zero.

(iv) Let $u, v$ be two vertices of $G$ of distance $m$, where $m \leq s+1$. Each shortest $(u, v)$-path has length $m$ and thus corresponds to a unique ordered representation

$$
u-v=s_{1}+s_{2}+\cdots+s_{m}
$$

with $s_{1}, \ldots, s_{m} \in S$. If some $s \in S$ appears more than once in this summation, then we can delete a pair of them (we are in $\mathbb{Z}_{2}$, so $s+s=0$ ) and find a shorter representation (and a shorter $(u, v)$-path), which does not exist. So $s_{1}, \ldots, s_{m}$ are distinct. Any other shortest $(u, v)$-path gives another ordered representation

$$
u-v=s_{1}^{\prime}+s_{2}^{\prime}+\cdots+s_{m}^{\prime},
$$

in which $s_{1}^{\prime}, \ldots, s_{m}^{\prime}$ are distinct by a similar argument, and we have $s_{1}+\cdots+s_{m}+$ $s_{1}^{\prime}+\cdots+s_{m}^{\prime}=0$. By linear independence of every $(2 s+2)$-subset of $S,\left(s_{1}^{\prime}, \ldots, s_{m}^{\prime}\right)$ is a permutation of $\left(s_{1}, \ldots, s_{m}\right)$. Therefore, the number of ordered representations of $u-v$ using $m$ members of $S$ is $m$ !, so the number of shortest $(u, v)$-paths in $G$ is also $m$ !, which is not more than $(s+1)$ !. 
(v) Without loss of generality, we may assume that $A \subseteq N(u)$. Every $a \in A$ can be written as $a=u+e_{i}$ for some $e_{i} \in S$. There is a set $B \subseteq S$ of size at least $d-|A|$ such that for every $e \in B, u+e \notin A$. For every $s$-subset $\left\{e_{i_{1}}, \ldots, e_{i_{s}}\right\}$ of $B$, we have a vertex $u+e_{i_{1}}+\cdots+e_{i_{s}}$ of distance $s$ from $u$. These vertices are all in $N_{s}^{A}(u)$ and are distinct, because of the linear independence of every $(2 s+2)$-subset of $S$. Hence we have

$$
\left|N_{s}^{A}(u)\right| \geq\left(\begin{array}{c}
d-|A| \\
s
\end{array}\right) \geq\left(\frac{d-|A|}{s}\right)^{s} \geq \frac{d^{s}}{(2 s)^{s}},
$$

where the last inequality follows from $d \geq 2(s+1) ! \geq 2|A|$.

(vi) By linear independence of every $2 s+2$ members of $S$, the distance between vertices 0 and $e_{1}+\cdots+e_{s+1}$ is at least $s+1$.

Theorem 1. Let s be a fixed positive integer denoting the speed of the robber. For every $n$, there exists a connected $n$-vertex graph with cop number $\Omega\left(n^{s / s+1}\right)$.

Proof. Take $k_{0}$ large enough so that $d=2^{k_{0}}$ satisfies $d \geq 2(s+1) !$ and $d^{s}>4(s+1) !(2 s)^{s}$. We may assume that $n>2^{1+k_{0}(s+1)}$. Let $k \geq k_{0}$ be the largest integer with $2^{1+k(s+1)} \leq n$, and let $n_{0}=2^{1+k(s+1)}$. By the way $k$ is defined, we have $n<2^{s+1} n_{0}$, so $n=\Theta\left(n_{0}\right)$. Let $G$ be the graph described above with parameters $k, s$. Let $m=(s+1)$ ! and let $q$ satisfy the equation $q d^{s}=2\left\lfloor\frac{d^{s}}{2(2 s)^{s}}\right\rfloor$. By Lemma 2, $G$ is a connected bipartite $d$-regular graph with $n_{0}=O\left(d^{s+1}\right)$ vertices and diameter larger than $s$. Also, for every two vertices $u, v$ of $G$ of distance at most $s+1$, there are at most $m$ distinct shortest $(u, v)$-paths, and for every vertex $u$ of $G$ and every subset $B$ of size at most $m$,

$$
\left|N_{s}^{B}(u)\right| \geq(d / 2 s)^{s} \geq q d^{s}
$$

Moreover, $q d^{s} / 2$ is an integer and

$$
q d^{s} / 2=\left\lfloor\frac{d^{s}}{2(2 s)^{s}}\right\rfloor \geq \frac{d^{s}}{4(2 s)^{s}}>m .
$$

Now by Lemma 1 , if the robber has speed $s$, then the cop number of $G$ is $\Omega\left(d^{s}\right)=$ $\Omega\left(n_{0}{ }^{s / s+1}\right)=\Omega\left(n^{s / s+1}\right)$. Let $G^{\prime}$ be the graph obtained by joining some vertex of $G$ to an endpoint of a path with $n-n_{0}$ vertices. It is easy to check that $G^{\prime}$ is a connected graph on $n$ vertices, whose cop number is the same as the cop number of $G$, which is $\Omega\left(n^{s / s+1}\right)$.

\section{Concluding remarks}

Following the notation of [10], let $f_{s}(n)$ be the maximum cop number of a connected $n$-vertex graph when the robber has speed $s$. It is well-known that $f_{1}(n)=\Omega(\sqrt{n})$ (the standard construction comes from the incidence graph of a projective plane, see, e.g., [12]). Meyniel conjectured that in fact $f_{1}(n)=\Theta(\sqrt{n})$ [4]. Frieze et al. [5] showed 
that $f_{s}(n)=\Omega\left(n^{\frac{s-3}{s-2}}\right)$. They also showed that when the robber can move through an unblocked path of arbitrary length in her turn, the cop number can be $\Omega(n)$. The second author conjectured that $f_{s}(n)=\Theta\left(n^{s / s+1}\right)$ for every $s$ [10]. In the present note we proved that $f_{s}(n)=\Omega\left(n^{s / s+1}\right)$, so the natural open question is to prove or disprove that $f_{s}(n)=O\left(n^{s / s+1}\right)$. This seems to be a difficult problem (even for the case $s=1$ the best known bound is $f_{1}(n) \leq n^{1-o(1)}$, which is far from the conjectured $O(\sqrt{n})$ bound), and the only general upper bound, given by Frieze et al., is the following: If $\alpha=1+s^{-1}$, then

$f_{s}(G) \leq n \alpha^{-(1-o(1)) \sqrt{\log _{\alpha} n}}$. Another interesting line of research is to study the maximum cop number of certain classes of graphs, e.g., random graphs - see [2, 8] for several results.

Acknowledgement. The authors thank Nick Wormald for many helpful discussions.

\section{References}

[1] N. Alon and J. Spencer, The probabilistic method, third ed., Wiley, 2008.

[2] B. Bollobás, G. Kun, and I. Leader, Cops and robbers in a random graph, arXiv:0805.2709v1 [math.CO].

[3] F. V. Fomin, P. A. Golovach, J. Kratochvíl, N. Nisse, and K. Suchan, Pursuing a fast robber on a graph, Theor. Comput. Sci. 411 (2010), no. 7-9, 1167-1181.

[4] P. Frankl, Cops and robbers in graphs with large girth and Cayley graphs, Discrete Appl. Math. 17 (1987), no. 3, 301-305.

[5] A. Frieze, M. Krivelevich, and P. Loh, Variations on cops and robbers, arXiv: 1004.2482v1 [math.CO].

[6] G. Hahn, Cops, robbers and graphs, Tatra Mt. Math. Publ. 36 (2007), 1-14.

[7] L. Lu and X. Peng, On Meyniel's conjecture of the cop number, submitted, 2009.

[8] T. Łuczak and P. Prałat, Chasing robbers on random graphs: Zigzag theorem, Random Struct. Alg. 37 (2010), 516 - 524.

[9] F. J. MacWilliams and N. J. A. Sloane, The theory of error correcting codes, North Holland, Amsterdam, 1997.

[10] A. Mehrabian, Lower bounds for cop number when robber is fast, arXiv:1007.1734v1 [math.CO].

[11] R. Nowakowski and P. Winkler, Vertex-to-vertex pursuit in a graph, Discrete Math. 43 (1983), no. 2-3, $235-239$.

[12] P. Prałat, When does a random graph have constant cop number?, Australas. J. Combin. 46 (2010), 285-296.

[13] A. Quilliot, Jeux et pointes fixes sur les graphes, Ph.D. thesis, Université de Paris VI, 1978.

[14] A. Scott and B. Sudakov, A new bound for the cops and robbers problem, arXiv:1004.2010v1 [math.CO]. 